Boletim Técnico da Escola Politécnica da USP Departamento de Engenharia de Construção Civil

\title{
A EXPRESSÃO GRÁFICA EM CURSOS DE ENGENHARIA: ESTADO DA ARTE E PRINCIPAIS TENDÊNCIAS
}

MORAES, Andréa Benício de CHENG, Liang-Yee 


\section{A EXPRESSÃO GRÁFICA EM CURSOS DE ENGENHARIA: ESTADO DA ARTE E PRINCIPAIS TENDÊNCIAS \\ MORAES, Andréa Benício de}

Universidade de Pernambuco - Escola Politécnica de Pernambuco

Rua Benfica, 455 - Recife, Pernambuco - andrea_benicio@uol.com.br

\section{CHENG, Liang-Yee}

Universidade de São Paulo - Escola Politécnica

Av. Prof. Almeida Prado, trav.2, nº 271 - Cid. Universitária, São Paulo - cheng@pcc.usp.br

\section{RESUMO:}

A tecnologia CAD com suas vantagens incomparáveis ao desenho através dos instrumentos tradicionais provocou a necessidade da reestruturação nos currículos das diversas universidades, no que concerne aos conteúdos programáticos e metodologias utilizadas no ensino de Desenho. Este trabalho apresenta um levantamento e uma análise das abordagens de ensino da Expressão Gráfica adotado pelos diversos cursos de engenharia do nosso país e no exterior. Objetiva o mesmo, contribuir para a modernização do ensino do desenho com a integração da computação gráfica e o uso das novas tecnologias na formação de profissionais para um mercado de trabalho cada dia mais exigente e globalizado.

Expressão gráfica, desenho técnico, geometria descritiva, geometria gráfica, ensino desenho engenharia

\section{ABSTRACT:}

The CAD technologies, with its incomparable advantages over the traditional drawing instruments has led the contents and the traditional teaching methodologies of Engineering Drawing inadequate, demanding a reformulation of the subject at several universities. This work present a survey and a analysis of the teaching approaches of Engineering Drawing adopted by several engineering courses of Brazil. It aims to contribute to the modernization of the teaching of the drawing by using new technologies in order to prepare the professionals for a more competitive market.

Graphic expression, technical drawing, descriptive geometry, graphic geometry, teaching drawing engineering

\section{INTRODUÇÃO}

Os avanços tecnológicos vêm trazendo grandes mudanças para a sociedade, e consequentemente para o ensino. Novas formas de trabalho foram geradas em função das possibilidades que as ferramentas computacionais podem oferecer, principalmente na área da engenharia gráfica. Por outro lado, no fomento à Qualidade do ensino no 
Brasil, as avaliações do MEC instigam as universidades a se aperfeiçoarem cada vez mais. Isto leva o ensino do Desenho, assim como a outras áreas de conhecimento, a uma verdadeira revolução no processo de reestruturação dos seus currículos. Podem-se perceber investimentos desprendidos na qualificação dos professores, pesquisas, e infraestrutura.

Diante destes desafios, pode-se dizer que o papel frente à introdução das novas tecnologias computacionais no ensino, ainda, não está claro, no sentido de contar com um plano pedagógico ou pelo menos, uma diretriz orientadora de como explorar melhor os recursos oferecidos.

No contexto do ensino da Representação Gráfica, muitos professores, na busca permanente de atualização metodológica, precisaram renovar as formas de abordagem de ensino, empregadas até então, em função do desenvolvimento da Gráfica Computacional. Novos conteúdos como os conceitos de modelamento geométrico e o desenvolvimento das habilidades relacionadas à ferramenta CAD vêm sendo adicionados às disciplinas de Desenho. Neste processo de transição encontramos uma grande heterogeneidade de metodologias utilizadas pelas universidades brasileiras proveniente de fatores geográficos, políticos, econômicos e sociais.

Este trabalho apresenta um levantamento e análise das formas de abordagens de ensino existentes, atualmente, considerando os novos recursos tecnológicos, metodologias e as novas exigências - em sentido amplo - do mercado de trabalho. Através de análise crítica e objetiva, pretende-se chegar a um retrato da situação do ensino da expressão gráfica no país, no intuito de contribuir para o esforço que vem sendo feito, instigando a discussão sobre o estado da arte das disciplinas de Teoria da Representação Gráfica em cursos de Engenharia.

\section{EVOLUÇÃO DO ENSINO DA EXPRESSÃO GRÁFICA}

"Desenho é criação do homem, seja pela necessidade de comunicar-se, de extravasar as suas angústias e alegrias, de se lançar ao mundo, de se organizar no espaço individual e coletivo, de estabelecer seus domínios, seja para registrar as suas idéias, e inegavelmente o Desenho tem a sua história na história da humanidade e, a cada dia, são estabelecidas novas conexões com as mais diversas áreas do conhecimento, merecendo atenção especial por toda a sua evolução e inclusão em diversos campos do conhecimento humano" (CAMPOS, 2000).

Até a revolução industrial o desenho era realizado de maneira que cada profissional se baseava empiricamente na sua experiência, não havendo um tratamento científico para a representação. Como o executor era também o projetista, o desenho servia apenas para registrar as idéias, sem a preocupação com a descrição completa do objeto, segundo VELASCO (1998).

As necessidades industriais de padronização para os objetos e produtos acarretaram numa separação mais nítida entre a concepção e a execução, gerando com isso a necessidade da comunicação entre as duas fases. Esta lacuna foi devidamente preenchida quando no final do século XVIII, o francês Gaspar Monge concebeu a técnica por ele denominada Geometria Descritiva, cuja representação de formas tridimensionais permite 
a descrição do objeto por qualquer um que venha a reproduzi-lo. Pela importância, este sistema, denominado Mongeano, passou a fazer parte do currículo escolar e, ainda hoje, é o método que constitui a base dos desenhos na engenharia (TRINCHÃO \& OLIVEIRA, 1998).

A disciplina Desenho, até a década de 40, era obrigatória no ensino médio. A Matemática se encarregava de dar o suporte geométrico que ela precisava. Esta importância, que se dava à disciplina Desenho, trazia um embasamento aos alunos que permitia um maior desenvolvimento dos conteúdos em nível universitário.

A Lei 4.024/61 integra o estudo das artes em uma única disciplina - Educação Artística, e torna exclusividade dos cursos colegiais e superiores as disciplinas Desenho Geométrico e Desenho Técnico. Em 71, a LDB 5692 torna obrigatória a inclusão da Educação Artística nos currículos plenos de $1^{\circ}$ e $2^{\circ}$ graus, e deixa de tratar o Desenho como disciplina, passando a entendê-lo como conteúdo relativo ao estudo das Artes e ao estudo da Matemática, da $5^{\mathrm{a}}$ a $8^{\mathrm{a}}$ série do $1^{\mathrm{o}}$ grau (CAMPOS, 2000). Isto, promoveu uma desvalorização ao ensino do desenho que fragmentou-se em: Desenho Geométrico, Educação Artística e Matemática.

Com a fragmentação do Desenho no ensino fundamental e médio, coube aos professores universitários suprirem esta defasagem, ministrando assuntos mais básicos em disciplinas existentes com ou sem nenhum aumento de carga horária ou a inserção de novas disciplinas, cujos conteúdos já deveriam ser familiarizados pelos alunos.

O processo de projeto na Engenharia utiliza a ferramenta Desenho para a concepção, comunicação e documentação das idéias, constituindo-se assim, em uma das principais ciências de auxílio à formação profissional do engenheiro. Permite o estudo do conceito de espaço e de seus atributos, tais como forma, dimensão e posição relativa. A habilidade de visão espacial (capacidade extremamente necessária ao engenheiro) é adquirida com o domínio da geometria e, não apenas, com a utilização dos métodos de construções gráficas.

O ensino de uma maneira geral passa por uma grande transformação. Em todas as áreas vem sofrendo alterações de metodologias, revisão de conteúdos somados às mudanças trazidas pelas facilidades do computador. "Revisar seus processos e repensar seus métodos em função das alternativas que o computador pode oferecer, consiste em se remover verdadeiros 'dogmas' tradicionais, muito difíceis de se derrubar (MEDINA, 1991).

Não se discute mais a implementação ou não do uso de Sistemas CAD nas universidades; mas como fazê-la e/ou efetivá-la. As Universidades pioneiras nesta mudança mostram através de sua experiência que, enquanto uma série de processos de representação ficou ultrapassada, a modelagem através do computador exige uma maior capacidade de visualização espacial, renovando a importância dos conceitos da Geometria Projetiva. É importante ressaltar, também, a necessidade do esboço que precede a elaboração do projeto e, consequentemente, o ensino do Desenho à Mão Livre. 


\section{METODOLOGIA}

O levantamento realizado, através de um questionário, foi complementado por uma pesquisa bibliográfica e o contato com professores e alunos. Os aspectos considerados foram: o planejamento da disciplina (conteúdo, carga horária, enfoque, atividades e seqüência dos conteúdos) e infra-estrutura (equipamentos disponíveis e relação com o número de alunos). A amostra constituída por 20 universidades, extraída de um universo de 93, permite-nos ter uma visão da situação atual do ensino.

Após codificar os dados resultantes das respostas do questionário, buscou-se sintetizálos de forma a facilitar o trabalho de análise bem como o de apresentação, visando clareza e aproveitamento.

As seqüências dos tópicos fornecidas pelas universidades foram analisadas porque podem refletir a "estratégia de ensino" da teoria de representação gráfica das diversas universidades. Através da identificação de seqüências características ou seqüências mais divergentes, de experiências isoladas, procura-se discutir a adequação em termos de tecnologia e formação de engenheiros.

Como as seqüências são muito diversificadas, é muito difícil identificar grupos de universidades que apresentam seqüências iguais através dos métodos mais comuns da matemática clássica. No entanto, seria mais fácil encontrar grupos de seqüências semelhantes, muito semelhantes, ou completamente diferentes.

Estes conceitos de "semelhança" entre dois elementos podem ser muito bem modelados através de uma relação nebulosa. Por essa razão, foram utilizados os conceitos da Teoria de Conjunto Nebuloso (Fuzzy Set Theory) para modelar e analisar as seqüências. O mesmo pode ser definido através de uma função de pertinência $\mu_{\tilde{A}}$ : $(x)$ cujo valor é definido no intervalo fechado [0,1]. Os valores extremos indicam uma completa exclusão (zero), ou completa inclusão (um) em um conjunto. E os valores entre 0 e 1, representam os graus de pertinência intermediários de um elemento com relação ao conjunto sendo que, quanto mais próximo de 1, maior será a pertinência. A relação de semelhança pode ser descrita como uma relação nebulosa, pois ao contrário de uma relação exata do tipo " $x$ é igual a $y$ ", expressa o quanto " $x$ é parecido com $y "$ (CHENG,1999).

A metodologia adotada para identificar as abordagens didáticas semelhantes em função da sequenciação dos tópicos foi baseada no método de agrupamento nebuloso e utilizado por COLLET et al (2000).

Inicialmente foi utilizado o conceito de grau de conformidade para definir o índice da relação de semelhanças entre as universidades, duas a duas, baseada na seqüência dos tópicos. Este índice de relação de semelhança entre as universidades corresponde ao grau de pertinência ou coeficiente da matriz de relação nebulosa de semelhança de seqüências de tópicos entre as universidades. Sendo assim, aplicando-se a expressão (1) para todas as combinações possíveis, obtemos uma matriz de relação nebulosa que expressa a semelhança entre as universidades. 


$$
\mu_{S_{m, n}}=\frac{1}{o} \sum_{k=1}^{o} \frac{\widetilde{a}_{k m} \wedge \widetilde{a}_{k n}}{\widetilde{a}_{k m} \vee \widetilde{a}_{k n}}
$$

Onde:

$\mu_{s m, n}=$ índice de semelhança dos programas entre 2 universidades $(\mathrm{m}, \mathrm{n})$;

$\tilde{a}_{k, m}=$ grau de pertinência de um determinado tópico $\mathrm{k}$ ocupar a posição inicial no programa da universidade $\mathrm{m}$;

$m, n=$ índices que representam as universidades; $m=1, \ldots, p$ e $n=1, \ldots, p$;

$k=$ índice que representa o tópico; $k=1, \ldots, o$;

$o=$ número de tópicos.

Para agrupar as universidades de acordo com o nível de semelhança dada na matriz nebulosa, considerando o aspecto da sequenciação dos tópicos das disciplinas de teoria da representação, utilizamos o método de corte de nível $\alpha$. O método de agrupamento por corte de nível $\alpha$ é um método simples onde se atribui um valor $\alpha \in[0,1]$, que expressa a exigência sobre o nível de semelhança entre os elementos a serem agrupados.

\section{RESULTADOS}

Dos resultados obtidos, verificam-se divergências entre os programas quanto ao número de disciplinas, às cargas horárias por disciplina e por curso e à posição das disciplinas e conteúdos no curso (MORAES \& CHENG, 2000).

Outros aspectos importantes, também, podem ser observados. Constata-se, por exemplo, que a grande maioria dos alunos que ingressaram na universidade não estudou desenho e os poucos que se beneficiaram com esta disciplina, podem ser, ainda, provenientes das Escolas Técnicas. Outro aspecto é o alto índice de qualificação dos professores de Desenho das universidades que responderam ao questionário. Este resultado pode até nem refletir a situação real do país, devido à estas universidades se concentrarem próximas de cursos de pós graduação na área.

Quanto ao uso do CAD, das 20 universidades consultadas, 16 já utilizam o computador nas aulas de Desenho. Entretanto, entre estas, 8 só começaram a utilizá-lo no ano 2000, representando uma mudança recente sem resultados definitivos (que permitam análise ou conclusões) desta aplicação.

Quanto aos conteúdos, estes vão desde uma breve revisão em desenho geométrico (que é parte do assunto a ser visto no ensino fundamental e ou médio), Sistema cilíndrico ortogonal (mongeano), Sistema cilíndrico ortogonal (axonometria), Sistema cilíndricooblíquo (cavaleira) e o Estudo do ponto, reta e plano (Sistema Cotado e Sistema Diédrico). Nas universidades que já utilizam as ferramentas CAD, podemos encontrar dois modelos básicos de abordagem: o seu uso logo na primeira disciplina de desenho; e a sua utilização nos períodos seguintes. Este último considera necessário, à aprendizagem do desenho por computador, um embasamento teórico inicial. Geralmente, os assuntos envolvidos são Vistas Ortográficas em sistema CAD 2D e Modelamento geométrico (CAD 3D). 


\section{ANÁLISE NEBULOSA DOS PROGRAMAS}

Tendo em vista que as seqüências dos conteúdos programáticos, muitas vezes, refletem a "estratégia de ensino", foi feita a análise de agrupamento das diferentes seqüências, adotadas pelas universidades, para identificar as principais abordagens didáticas existentes, investigar suas semelhanças e diferenças e verificar sua adequação às exigências atuais, em termos de tecnologias e, principalmente, necessidades do conhecimento do engenheiro.

Para uma determinada disciplina, as razões que levam as universidades a trabalharem ou não com determinados tópicos e de acordo com uma seqüência específica, podem ser em virtude da abordagem didática do professor, do pré-requisito de um tópico sobre o outro, dos recursos disponíveis para implementação do sistema CAD ou simplesmente da ênfase ou modalidade do curso.

Procuramos aplicar a teoria dos sistemas nebulosos para a análise de agrupamento dos programas das disciplinas de Teoria de Representação Gráfica, adotadas pelas diversas universidades, considerando os tópicos trabalhados e suas respectivas seqüências de apresentação, com o objetivo de identificar, com o auxílio de bibliografias, professores e alunos, as principais abordagens didáticas existentes, investigar suas semelhanças e diferenças e verificar sua adequação às exigências atuais, em termos de tecnologias e, principalmente, a formação do engenheiro.

As seqüências foram obtidas com base na ordem apresentada nas respostas dos questionários, na tabela de conteúdos. Apesar da grande variedade de tópicos e abordagens diferentes para facilitar a análise, foram estabelecidos 7 tópicos principais, selecionados por sua abrangência e freqüência nas respostas: Sistema cilíndrico ortogonal (mongeano); Sistema cilíndrico ortogonal (axonometria); Sistemas cilíndricooblíquo (cavaleira); Estudo do ponto, reta e plano (Sistema Cotado); Estudo do ponto, reta e plano (Sistema Diédrico); Vistas Ortográficas (CAD 2D); Modelamento geométrico (CAD 3D).

A partir dos questionários respondidos, foi montada a tabela 1 com os tópicos acima e todas as seqüências fornecidas. Os valores referem-se a ordem em que cada tópico ocupa dentro do programa. As linhas referem-se aos tópicos acima citados e cada coluna representa as respostas obtidas da universidade identificada por uma letra. Os espaços com valores nulos indicam que os tópicos não foram citados ou informados. Como as universidades I e K não forneceram a ordem dos tópicos, não fizeram parte desta análise.

Tabela 1 Seqüência dos tópicos de cada universidade

\begin{tabular}{|l|c|c|c|c|c|c|c|c|c|c|c|c|c|c|c|c|c|c|c|}
\hline \multicolumn{1}{|c}{ TóPICOSIUNIVERSIDADES } & A & B & C & D & E & F & G & H & J & L & M & N & O & P & Q & R & S & T \\
\hline $\begin{array}{l}\text { Sistema cilíndrico ortogonal (mongeano) } \\
\text { Sistema cilíndrico ortogonal (axonometria) }\end{array}$ & 1 & 1 & 1 & 6 & 1 & 3 & 2 & 2 & 1 & 1 & 1 & 1 & 2 & 1 & 1 & 2 & 3 & 3 \\
\hline $\begin{array}{l}\text { Sistemas cilíndrico-oblíquo (cavaleira) } \\
\text { Estudo do ponto, reta e plano (Sistema Cotado) }\end{array}$ & 2 & 2 & 2 & 3 & 2 & 2 & 5 & 4 & 2 & 2 & 3 & 3 & 3 & 4 & 2 & 4 & 2 & 5 \\
\hline $\begin{array}{l}\text { Estudo do ponto, reta e plano (Sistema Diédrico) } \\
\text { Vistas Ortográficas (CAD 2D) }\end{array}$ & 0 & 3 & 3 & 4 & 0 & 1 & 1 & 3 & 0 & 0 & 2 & 0 & 1 & 3 & 0 & 3 & 4 & 6 \\
\hline Modelamento geométrico (CAD 3D) & 0 & 4 & 5 & 5 & 3 & 4 & 3 & 1 & 0 & 0 & 0 & 2 & 4 & 0 & 0 & 1 & 1 & 1 \\
\hline
\end{tabular}


Da tabulação inicial das respostas do questionário, observa-se que, de um modo geral, a seqüência dos conteúdos é diferenciada mesmo sem a utilização de um sistema CAD e, exceto em uma universidade, os tópicos relacionados aos mesmos foram alocados para as etapas finais de curso.

Apenas dois tópicos foram citados por todas as universidades: Sistema cilíndrico ortogonal (mongeano) e Sistema cilíndrico ortogonal (axonometria); ao contrário dos outros tópicos que deixaram de ser citados por uma ou outra universidade. Isto pode ser justificado pelo fato destes dois tópicos serem a base da representação gráfica, onde seus conceitos são utilizados de uma forma muito ampla na Engenharia, como no Desenho Técnico que, geralmente, utiliza as projeções ortogonais para representar um objeto através das suas vistas ortográficas e da sua perspectiva axonométrica.

Os tópicos 3, 4, 5, e 6 (Sistemas cilíndrico-oblíquo (cavaleira), Estudo do ponto, reta e plano (Sistema Cotado), Estudo do ponto, reta e plano (Sistema Diédrico), Vistas Ortográficas (CAD 2D)) não aparecem em 1/3 dos programas das disciplinas.

Quanto à seqüência, o tópico que aparece em maior número de vezes em primeira posição (10 universidades - 56\% do total), é Sistema cilíndrico ortogonal (mongeano). Este arranjo é normalmente encontrado nos livros de desenho técnico mais tradicionais o que pode ter contribuído para colaborar na adoção de uma programação semelhante nas universidades. O tópico Sistema cilíndrico ortogonal (axonometria) é o que caracteristicamente ocupa a segunda posição ( 9 vezes - 50\% do total). A maioria das ementas que iniciam com representação de sólidos em vistas ortogonais apresenta a representação do sólido em perspectiva axonométrica como o segundo tópico do programa.

Quanto aos Sistemas cilíndrico-oblíquo - utilizados no sistema de representação da perspectiva cavaleira - embora considerado um sistema versátil, tem a característica de deformar o objeto representado e por esta razão não ser tão utilizado quanto à perspectiva axonométrica. Em seis universidades (33\% do total), este tópico não foi citado. Concordando com este fato, em 1998 numa pesquisa da universidade do Texas para a criação de um novo currículo para o ensino da Geometria Gráfica para cursos de engenharia, BARR (1998) verificou que este assunto, também não foi citado, dentre os mais importantes pelos professores consultados.

Os tópicos referentes ao sistema $\mathrm{CAD}$, aparecem quase sempre nas etapas finais dos programas. O tópico Modelamento de Geométrico (CAD 3D) foi citado 7 vezes (39\% do total). Apenas uma universidade o posicionou na fase inicial do programa. Este assunto ainda é pouco explorado nas universidades, pois requer sistemas CAD mais poderosos e consequentemente investimentos mais elevados em hardware e software.

$\mathrm{Na}$ prática, constata-se que trabalhando inicialmente com sólidos tridimensionais para depois estudar seus elementos isoladamente ou modelá-lo no computador, pode-se promover um maior interesse pela disciplina, além de amenizar as dificuldades do processo de desenvolvimento da habilidade da visualização espacial.

Este fato pode ser verificado quando examinamos a tabela 1, onde apenas 4 das universidades iniciam o assunto da disciplina com o estudo do ponto, reta e plano 
através do sistema diédrico. Também na tabela 1, verifica-se que o tópico projeções cotadas ocupa as mais diferentes posições, embora nunca seja apresentado no início dos cursos. Segundo VILLAROUCO (1993), a projeção de elementos em apenas um plano, juntamente com as respectivas cotas para a solução de problemas gráficos, apresenta-se como um sistema cujas operações tornam-se mais simples que no sistema diédrico (Mongeano).

Para a aplicação da Teoria dos Sistemas Nebulosos, relacionamos as universidades e os tópicos, ponderando os valores dos coeficientes pelo número total de tópicos de cada universidade. O coeficiente $\tilde{a}_{i j}$ da matriz de relações nebulosas $\tilde{A}$ assim calculada representa o grau de pertinência de um determinado tópico $i$ ocupar a posição inicial no programa da disciplina da universidade $j$.

Feito isso, utilizamos o conceito de grau de conformidade entre 2 elementos apresentado por COLLET et al (2000) para estabelecer o índice de semelhança entre 2 universidades $m$ e $n$, conforme mostra a equação (1). Em outras palavras, o índice de semelhança $\mu s_{m, n}$ dos programas de 2 universidades $(m, n)$ é definido pela relação entre a interseção e a união de todas as suas características (no caso do problema em estudo, o grau de pertinência de um determinado tópico ocupar a posição inicial nos respectivos programas).

Repetindo o cálculo para todos os pares de universidades $(m, n)$, obtêm-se uma matriz de relação nebulosa da semelhança entre as universidades onde pode-se observar que a semelhança entre as seqüências das universidades apresentaram o valor 1 para duas universidades, A e J, caracterizando programas idênticos. O resultado está de acordo com a tabela 1: o programa destas universidades apresentam apenas 4 dos 7 tópicos citados e os conteúdos são abordados na seguinte ordem: Sistema cilíndrico ortogonal (mongeano), Sistema cilíndrico ortogonal (axonometria), Estudo do ponto, reta e plano (Sistema Cotado), Vistas Ortográficas (CAD 2D).

As semelhanças entre as seqüências dos conteúdos é observada no dendograma da Figura 1, resultante da aplicação do método de agrupamento nebuloso por corte de nível $\alpha$ na matriz da relação nebulosa. $O$ dendograma oferece uma visualização gráfica do agrupamento entre as universidades com seqüências semelhantes. No eixo das abcissas estão as universidades e nas ordenadas está uma escala de valores referentes ao nível de semelhança.

Do dendograma, podemos notar que para níveis de corte variando entre 1 e 0,7 , grande parte das universidades vão se agrupando gradualmente, formando 3 grupos principais que se fundem em 2 grupos grandes para valor de corte de 0,7. Com grau de aproximadamente 0,5 , todas as universidades pertencem a apenas um grupo.

O maior valor encontrado na matriz, abaixo de 1 , foi $\alpha=\mathbf{0 , 9 1}$, onde as universidades $\mathbf{B}$ e O se agruparam. Isto pode ser comprovado ao se observar na tabela 1. Ambos os programas possuem os mesmos tópicos. Os tópicos 4, 5, 6 e 7, ocupam a mesma posição nas duas universidades, ou seja, Estudo do ponto, reta e plano (Sistema Diédrico) na quarta posição, seguido de: Estudo do ponto, reta e plano (Sistema Cotado), Sistema cilíndrico ortogonal (mongeano) e Modelamento geométrico (CAD 3D). 


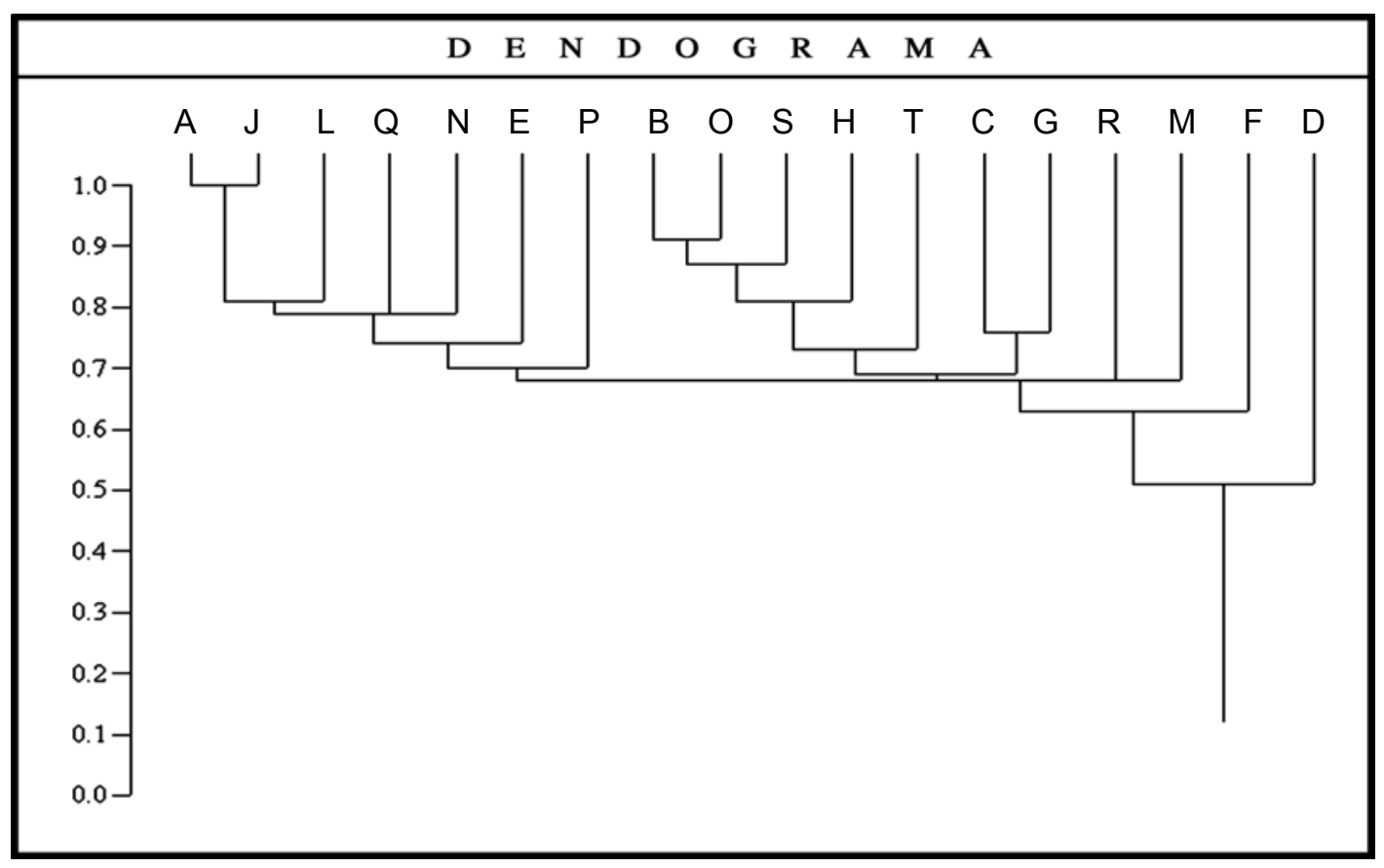

Figura 1 Dendograma resultante do Agrupamento Nebuloso por Corte de Nível $\alpha$

No grupo $\{\mathbf{B}, \mathbf{O}\}$, a posição dos 3 primeiros tópicos está diferente (Sistema cilíndrico ortogonal (mongeano), Sistema cilíndrico ortogonal (axonometria), Sistemas cilíndricooblíquo (cavaleira)). A universidade B inicia seu programa com apresentação de vistas ortogonais, e a universidade $\mathbf{O}$ inicia seu programa com o tópico Sistemas cilíndricooblíquo (cavaleira) seguido de Sistema cilíndrico ortogonal (mongeano), assim como outras duas instituições. A razão disso pode ser o fato de que muitos alunos iniciantes em Geometria Gráfica considerem que a perspectiva cavaleira, aplicação mais usual da projeção cilíndrica-oblíqua, tenha uma construção mais simples que as demais perspectivas, considerando seu processo, sem o auxílio do computador.

Segundo COSTA (1988), a perspectiva tem a característica de ser a forma mais elucidativa de representação, por "se assimilar a imagem visual que o observador recebe quando olha para o objeto". Somando-se à facilidade de construção, podemos aí encontrar a justificativa para sua prévia utilização.

Aplicando o conceito do corte de nível alfa, se diminuirmos a exigência do nível de semelhança para próximo valor $\alpha=\mathbf{0 , 8 7}$, a universidade $\mathbf{S}$ soma-se ao grupo $\{\mathbf{B}, \mathbf{O}\}$. Os tópicos em posições idênticas são: Estudo do ponto, reta e plano (Sistema Cotado), Vistas Ortográficas (CAD 2D) e Modelamento geométrico (CAD 3D), em $5^{\mathrm{a}}, 6^{\mathrm{a}}$ e $7^{\mathrm{a}}$ posições respectivamente. Todos os outros tópicos, também, estão presentes mas em ordens diferentes.

Diminuindo mais o nível de semelhança temos o valor $\alpha=\mathbf{0 , 8 1}$, onde mais uma universidade, $\mathbf{H}$, se insere ao grupo $\{\mathbf{B}, \mathbf{O}\}$. E a universidade $\mathbf{L}$, ao grupo $\{\mathbf{A}, \mathbf{J}\}$. O que diferencia mais nestes dois grupos é o fato de que o grupo $\{\mathbf{A}, \mathbf{J}, \mathbf{L}\}$, trabalha com um programa compacto de no máximo quatro dos sete tópicos, em contrapartida ao grupo $\{\mathbf{B}, \mathbf{O}, \mathbf{S}, \mathbf{H}\}$ de programas mais extensos, todos com mais de 6 tópicos e com a 
seqüenciação começando com desenho a instrumento e terminando com uso de CAD, em etapas bem distintas.

Diminuindo mais ainda a exigência do nível de semelhança para próximo valor $\alpha=\mathbf{0 , 7 9}$, as universidades $\mathbf{N}$ e $\mathbf{Q}$ somam-se ao grupo $\{\mathbf{A}, \mathbf{J}, \mathbf{L}\}$ que permanece com a característica de conter no máximo quatro dos tópicos, tendo em comum: Sistema cilíndrico ortogonal (mongeano), Sistema cilíndrico ortogonal (axonometria) e Vistas Ortográficas (CAD 2D). As características destes programas compactos são: iniciar o tópico Sistema cilíndrico ortogonal (mongeano); seguir com Sistema cilíndrico ortogonal (axonometria) (com exceção da universidade $\mathbf{N}$ ) e terminar com tópicos de $\mathrm{CAD}$, sendo que, o Modelamento geométrico (CAD 3D) só aparece no programa da universidade $\mathbf{Q}$.

Comparando os grupos $\{\mathbf{A}, \mathbf{J}, \mathbf{L}, \mathbf{N}, \mathbf{Q}\}$ e $\{\mathbf{B}, \mathbf{O}, \mathbf{S}, \mathbf{H}\}$, podemos constatar um fato curioso: no primeiro, os programas são compactos e nenhum deles incluem o tópico Sistema cilíndrico-oblíquo (cavaleira), mas incluem os tópicos referentes ao sistema CAD (na maioria, trabalha pelo menos com CAD 2D). Já no segundo grupo, o tópico Sistema cilíndrico-oblíquo (cavaleira) aparece nas primeiras posições e os tópicos que utilizam o sistema $\mathrm{CAD}$, aparecem nas últimas posições. O que nos parece é que, devido a sua importância secundária, diante do tempo limitado de um programa compacto, a introdução do $\mathrm{CAD}$ tem custado a exclusão do tópico Sistema cilíndrico-oblíquo (cavaleira).

O próximo nível abaixo é $\alpha=\mathbf{0 , 7 7}$. Os grupos $\{\mathbf{A}, \mathbf{J}, \mathbf{L}, \mathbf{N}, \mathbf{Q}\}$ e $\{\mathbf{B}, \mathbf{O}, \mathbf{S}, \mathbf{H}\}$ continuam sem alterações, mas $\{\mathbf{C}, \mathbf{G}\}$ formam um novo grupo, que caracteriza-se por não conter o tópico Modelamento geométrico (CAD 3D) e coincidir Estudo do ponto, reta e plano (Sistema Cotado), na quarta posição e Vistas Ortográficas (CAD 2D) na sexta e última posição.

Podemos representar os agrupamentos através de diagramas de círculos, onde cada círculo representa uma universidade que ligada à outra, formam um grupo ou conjunto de elementos semelhantes.
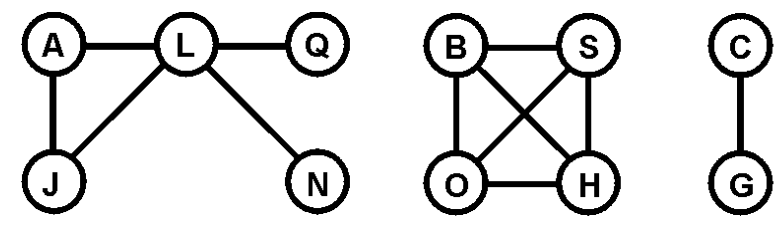

Figura 2 - Diagrama de círculos $\alpha=0,77$

O diagrama de círculos para $\alpha=\mathbf{0 , 7 7}$ (Figura 2) também mostra que a relação interna entre os elementos nos grupos compacto e extenso são diferentes. No caso do grupo compacto pode-se identificar a universidade $\mathrm{L}$ como o elemento representativo do grupo, pois, é o único que está vinculado a todos os elementos do mesmo grupo. Ao invés disso, no grupo extenso, todos os elementos se relacionam com todos, mostrando maior homogeneidade.

Ao diminuirmos o nível para $\alpha=\mathbf{0 , 7 4}$, a universidade $\mathbf{E}$ se insere ao grupo de ementas compactas, mas o que difere do grupo inicial $\{\mathbf{A}, \mathbf{J}, \mathbf{L}, \mathbf{N}, \mathbf{Q}\}$ é que seu programa, 
embora compacto, não utiliza os sistemas CAD. No nível $\alpha=\mathbf{0 , 7 3}$, a universidade $\mathbf{T}$ se insere no grupo $\{\mathbf{B}, \mathbf{O}, \mathbf{S}, \mathbf{H}\}$. Nesta universidade podemos observar que o sistema CAD é abordado ao longo do curso, tendo Vistas Ortográficas (CAD 2D) logo após o tópico Sistema cilíndrico ortogonal (mongeano) e Modelamento geométrico (CAD 3D) logo após os tópicos Sistema cilíndrico ortogonal (axonometria) e Sistema cilíndrico-oblíquo (cavaleira), com a seguinte seqüência:

\section{$2 \mathrm{D}$ (tradicional) $\rightarrow$ 2D (CAD) $\rightarrow$ 3D (tradicional) $\rightarrow$ 3D (CAD)}

No nível $\alpha=\mathbf{0 , 7 1}$, a universidade $\mathbf{P}$ é inserida no grupo $\{\mathbf{A}, \mathbf{J}, \mathbf{L}, \mathbf{N}, \mathbf{Q}, \mathbf{E}\}$. Esta, com cinco tópicos, inclui Sistema cilíndrico-oblíquo (cavaleira) em $3^{\mathrm{a}}$ posição, mas deixa de fora o Estudo do ponto, reta e plano (Sistema Diédrico) e o Modelamento geométrico (CAD 3D). O grupo $\{\mathbf{A}, \mathbf{J}, \mathbf{L}, \mathbf{N}, \mathbf{Q}, \mathbf{E}, \mathbf{P}\}$ trabalha com os programas do tipo:

\section{1- Vistas $\rightarrow$ Perspectivas ou \\ 2- Vistas $\rightarrow$ Perspectivas $\rightarrow$ CAD}

Com grau de semelhança $\alpha=\mathbf{0 , 7 0}$, os grupos $\{\mathbf{B}, \mathbf{O}, \mathbf{S}, \mathbf{H}, \mathbf{T}\}$ e $\{\mathbf{C}, \mathbf{G}\}$.se fundem. Resultando assim, 2 grandes grupos: $\{\mathbf{A}, \mathbf{J}, \mathbf{L}, \mathbf{Q}, \mathbf{N}, \mathbf{E}, \mathbf{P}\}$ e $\{\mathbf{B}, \mathbf{O}, \mathbf{S}, \mathbf{H}, \mathbf{T}, \mathbf{C}, \mathbf{G}\}$, ilustrados na figura 3. Suas características são bem peculiares, respectivamente, programas compactos e extensos. Por outro lado, também apresentam características em comuns: exceto alguns programas compactos que não introduziram o desenho com sistemas $\mathrm{CAD}$, a divisão de tópicos em desenho instrumental (tradicional) e desenho com CAD é bastante nítida, sendo que o último é deixado para a parte final da disciplina.
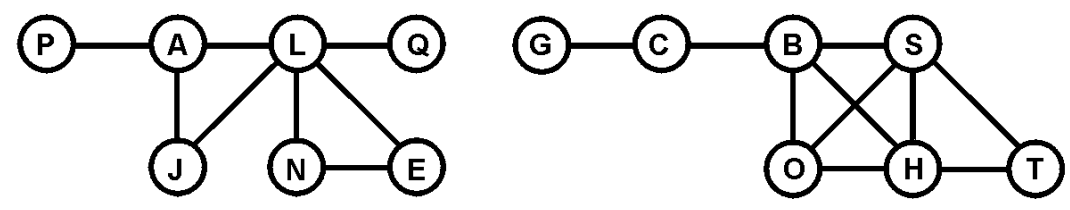

Figura 3 - Diagrama de círculos $\alpha=0,70$

Ao mesmo tempo em que os resultados mostram que existem dois grupos de universidades, sendo seus programas compactos e extensos, não encontramos relação destes grupos com a carga horária determinada para estes cursos, como podemos verificar na tabela 2 :

Tabela 2 Carga horária de cada universidade

CARGA HORÁRIA /

UNIVERSIDADES

\begin{tabular}{|c|c|c|c|c|c|c|c|c|c|c|c|c|c|c|c|c|c|}
\hline A & $\mathbf{B}$ & $\mathbf{C}$ & $\mathbf{D}$ & $\mathbf{E}$ & $\mathbf{F}$ & $\mathbf{G}$ & $\mathbf{H}$ & $\mathbf{J}$ & $\mathbf{L}$ & $\mathbf{M}$ & $\mathbf{N}$ & $\mathbf{O}$ & $\mathbf{P}$ & $\mathbf{Q}$ & $\mathbf{R}$ & $\mathbf{S}$ & $\mathbf{T}$ \\
\hline 150 & 36 & 120 & 60 & 96 & 135 & 150 & 240 & 60 & 120 & 60 & 135 & 135 & 165 & 240 & 120 & 120 & 180 \\
\hline
\end{tabular}

$\mathrm{CH}$ :soma das cargas horárias das disciplinas de Teoria da Representação

Os dois grandes grupos se fundem e incluem a universidade $\mathbf{R}$ no nível $\alpha=\mathbf{0 , 6 9}$, e a universidade $\mathbf{M}$ no nível $\alpha=\mathbf{0 , 6 8}$. A característica destas universidades é não trabalhar com sistemas CAD, ter o programa reduzido mas incluir o Sistema cilíndrico-oblíquo (cavaleira). No nível $\alpha=\mathbf{0 , 6 4}$, entra a universidade $\mathbf{F}$, que caracteriza-se por ter um programa extenso, mas trabalha apenas com instrumento tradicional, sem nenhum tópico relacionado ao CAD. O grande grupo, $\{\mathbf{A}, \mathbf{J}, \mathbf{L}, \mathbf{Q}, \mathbf{N}, \mathbf{E}, \mathbf{P}, \mathbf{B}, \mathbf{O}, \mathbf{S}, \mathbf{H}, \mathbf{T}, \mathbf{C}, \mathbf{G}, \mathbf{T}, \mathbf{R}$, 
$\mathbf{M}, \mathbf{F}$, agora engloba todas as universidades, com exceção da universidade $\mathbf{D}$, que só se insere no nível $\alpha=\mathbf{0 , 5 1}$.

O programa da universidade $\mathbf{D}$ possui uma característica própria e mostra a preocupação com a integração maior dos tópicos de CAD no curso de Desenho. A programação da universidade D, introduzida em 1999, apresenta abordagem totalmente distinta das demais, com uma inversão radical iniciando o curso com os tópicos de Modelamento geométrico (CAD 3D) e finalizando com o. Sistema cilíndrico ortogonal (mongeano).

De fato, das 18 universidades consideradas no estudo, 7 lidam com Modelamento geométrico (CAD 3D), dos quais 6 universidades colocam o tópico como o último a ser trabalhado. Esta posição concorda com os autores citados nos capítulos anteriores, que consideram necessário um embasamento teórico inicial à aprendizagem do desenho por computador.

$\mathrm{Na}$ abordagem da universidade $\mathbf{D}$, a inovação consiste em, uma vez feita a iniciação com o embasamento das técnicas de esboço e metodologia de projetos, o aluno passa a trabalhar com o modelamento geométrico no computador com recursos computacionais que facilitam a manipulação e visualização espacial, os tópicos caminham na direção da fundamentação teórica para chegar no final, representação $2 \mathrm{D}$ em vistas ortográficas e desenhos técnicos propriamente ditos, assistida pelos recursos de geração automática de vistas a partir de modelos de sólidos. O fluxo de trabalho é extremamente lógico e similar ao procedimento moderno de projeto de engenharia. Esta abordagem didática é acompanhada e avaliada através dos resultados do Mental Rotations Teste, ou MRT (MAFALDA et al, 1999) a qual, por ser uma experiência ainda incipiente, não forneceu dados que permitam apontar os resultados efetivos.

\section{CONSIDERAÇÕES FINAIS}

Da tabulação inicial das respostas do questionário, observa-se que, de um modo geral, a seqüência dos conteúdos é diferenciada mesmo sem a utilização de um sistema CAD. Entretanto, as vistas ortográficas e a projeção axonométrica são os tópicos comuns a todos os programas analisados. Em todas as instituições, exceto em uma, os tópicos relacionados aos sistemas $\mathrm{CAD}$ foram alocados para as etapas finais de curso.

Nos resultados encontrados, percebe-se que há dois grupos grandes e bem definidos de universidades, com características bem peculiares, cujos programas são, respectivamente, compactos e extensos. Já uma das universidades, apresenta uma abordagem totalmente distinta das demais. Estas experiências vivenciadas nos permitem discutir os méritos das diferentes abordagens de ensino, que podem no futuro, servir de parâmetro para novas reformulações no ensino da expressão gráfica.

Tendo em vista os resultados obtidos, acreditamos que o objetivo deste trabalho foi alcançado, em especial, no sentido de estarmos divulgando informações que visam, a partir do intercâmbio e troca de experiências, o aprimoramento das metodologias de ensino, diante das novas tecnologias e conceitos. Com a análise feita, será possível, em uma próxima etapa, a elaboração de uma proposta metodológica adequada à realidade social de cada universidade e, que sobretudo, objetive a necessidade da engenharia nacional e global. 


\section{REFERÊNCIAS}

BARR, R. E. Planning the EDG curriculum for the $21^{\text {st }}$ century: a proposed team effort. Engineering Design Graphics Journal, v.63, n.2, p.4, 1999.

CAMPOS, A. R. A. O estado do Desenho no ensino oficial brasileiro. In: CONGRESSO INTERNACIONAL DE ENGENHARIA GRÁFICA NAS ARTES E NO DESENHO, 3.; SIMPÓSIO NACIONAL DE GEOMETRIA DESCRITIVA E DESENHO TÉCNICO, 14., Ouro Preto, 2000. Anais. Ouro Preto, Graphica, 2000.

CHENG, L. Y. A teoria de sistema nebuloso e as informações subjetivas de conforto ambiental. In: ENCONTRO NACIONAL DE TECNOLOGIA APLICADA À CONSTRUÇÃO, 1999. Anais.

COLLET, V. G.; CHENG, L. Y.; PETRECHE, J. R. D. Análise nebulosa das imagens de fachada. In: CONGRESSO INTERNACIONAL DE ENGENHARIA GRÁFICA NAS ARTES E NO DESENHO, 3.; SIMPÓSIO NACIONAL DE GEOMETRIA DESCRITIVA E DESENHO TÉCNICO, 14., Ouro Preto, 2000. Anais. Ouro Preto, Graphica, 2000.

COSTA, M. D; COSTA, A. P. A. V. Geometria gráfica tridimensional. v.1, Recife, Universitária, 1988.

MAFALDA, R., et al. Avaliação da eficácia da reestruturação dos cursos de desenho para engenharia na Escola Politécnica da Universidade de São Paulo. In: CONGRESSO BRASILEIRO DE ENSINO NA ENGENHARIA, 27., Natal, 1999. Anais. Natal, Cobenge, 1999.

MEDINA, A. C. Experiências no ensino de desenho por computador. In: SIMPÓSIO NACIONAL DE REPRESENTAÇÃO GRÁFICA, GEOMETRIA DESCRITIVA E DESENHO TÉCNICO, 10., Salvador, 1991. Anais. Salvador, Graphica, 1991.

MORAES, A. B.; CHENG, L. Y. O estado da arte das disciplinas de desenho para cursos de engenharia. In: CONGRESSO BRASILEIRO DE ENSINO DE ENGENHARIA, 28., Ouro Preto, 2000. Anais. Ouro Preto, Cobenge, 2000.

TRINCHÃO, G. C.; OLIVEIRA, L. R. O. A História contada a partir do desenho. In: CONGRESSO INTERNACIONAL DE ENGENHARIA GRÁFICA NAS ARTES E NO DESENHO, 2.; SIMPÓSIO NACIONAL DE GEOMETRIA DESCRITIVA E DESENHO TÉCNICO, 13., Feira de Santana, 1998. Anais. Feira de Santana, Graphica, 1998. p 156.

VELASCO, A. A informática no ensino de desenho técnico. São Paulo, 1998. P. (Trabalho acadêmico). 